\title{
Determination of Triclocarban, Triclosan and Methyl-Triclosan in Environmental Water by Silicon Dioxide/Polystyrene Composite Microspheres Solid-Phase Extraction Combined with HPLC-ESI-MS
}

\author{
Yinan Wang ${ }^{1,2}$, Pengfei $\mathrm{Li}^{1,3}$, Yan Liu ${ }^{1}$, Bing Chen ${ }^{1}$, Jinyu $\mathrm{Li}^{1}$, Xikui Wang ${ }^{{ }^{*}}$ \\ ${ }^{1}$ School of Chemical Engineering, Shandong Polytechnic University, Jinan, China \\ ${ }^{2}$ School of Biological Science and Technology, Beijing Forestry University, Beijing, China \\ ${ }^{3}$ Jinan Water Group Company Limited, Jinan, China \\ Email: "talis.bachmann@ut.ee
}

Received July 2013

\begin{abstract}
This paper developed a sensitive and efficient analytical method for triclocarban (TCC), triclosan (TCS) and Methyl-triclosan (MTCS) determination in environmental water, which involves enrichment by using silicon dioxide/polystyrene composite microspheres solid-phase extraction and detection with HPLCESI-MS. The influence of several operational parameters, including the eluant and its volume, the flow rate and acidity of water sample were investigated and optimized. Under the optimum conditions, the limits of detection were $1.0 \mathrm{ng} / \mathrm{L}, 2.5$ and $4.5 \mathrm{ng} / \mathrm{L}$ for TCC, TCS, and MTCS, respectively. The linearity of the method was observed in the range of $5-2000 \mathrm{ng} / \mathrm{L}$, with correlation coefficients $\left(\mathrm{r}^{2}\right)>.99$. The spiked recoveries of TCC, TCS and MTCS in water samples were achieved in the range of $89.5 \%-96.8 \%$ with RSD below $5.7 \%$. The proposed method has been successfully applied to analyze real water samples and satisfactory results were achieved.
\end{abstract}

Keywords: Triclocarban; Triclosan; Solid-Phase Extraction; Silicon Dioxide/Polystyrene Composite Microspheres; HPLC-ESI-MS/MS

\section{Introduction}

Over the past decades, increasing residues of pharmaceuticals and personal care products (PPCPs) have been detected in the aquatic environment. Hundreds of tones of these compounds are dispensed in communities every year. The continuous input of such compounds may affect water quality and potentially affect the ecosystem and human health (Halden \& Paull, 2005; Ahn et al., 2008; Chalew \& Halden, 2009). Triclocarban (N-(4-chlorophenyl)-N-(3,4-dichlorophenylurea, TCC) and triclosan (5-chloro -2-(2,4-dichloro-phenoxy)-phenol, TCS) are both antimicrobials that are added to a wide range of household and personal-care products, such as shampoos, soaps, creams, mouthwash and toothpaste. However, TCC has harmful effects on humans and other animals because it increases methemoglobinemia (Ponte et al., 1974). TCC exposure enhances the estradiol-dependent or testos-terone-dependent activation of the estrogen receptor-responsive and the androgen receptorresponsive gene expression in human ovary cells (Ahn et al., 2008). Moreover, TCC affects the transcription of genes that respond to the thyroid hormone in frog and rat cells (Hinther et al., 2011). Bioaccumulation studies have shown that TCC accumulates in algae (Coogan et al., 2007) and snails (Coogan et al., 2008). Similarly, TCS was found to be acutely toxic to a number of aquatic organisms, particularly for algae species, and was recently shown to the modulate thyroid function of amphibians at concentrations as low as .15 $\mu \mathrm{g} / \mathrm{L}$ (Orvos et al., 2002).

"Corresponding author.
In addition, triclosan has been shown to phototransform into chlorinated dibenzodioxins (Mezcua et al., 2004). Methyl-triclosan (MTCS) is a metabolite of TCS. It is more lipophilic and environmentally persistent (Chu \& Metcalfe, 2007; Coogan et al., 2007), suggesting its relatively high bioaccu-mulation potential in aquatic organisms (Coogan et al., 2007).

The potential risk to the environment and human health generated by TCC, TCS and MTCS is currently drawing considerable attention worldwide. Therefore, it is very essential to establish simple, sensitive and reliable analytical method to determine these compounds in various environmental water samples for safety evaluation.

Generally, a sample enrichment and clean-up procedure are often needed prior to the instrumental determination because these pollutants are present at very low levels in environmental water samples ( $\mu \mathrm{g} / \mathrm{L}$ or less). Many sample pretreatment methods, such as liquid-liquid extraction (LLE) (Canosa et al., 2007; Sun et al., 2012), dispersive liquid-liquid microextraction (DLLME) (Guo et al., 2009; Zhao et al., 2011), solid-phase extraction (SPE) (Halden \& Paull, 2004; Sapkota et al., 2007), solid-phase micro-extraction (SPME) (Montes et al., 2005), hollow fiber assisted liquid-phase microextraction (HF-LPME) (Zhao et al., 2007), and stir bar sorptive extraction (SBSE) (Kawaguchi et al., 2008) have been used for the enrichment of TCS and TCC.

Traditional LLE is widely used as a sample preparation technique by several authors (Liska et al., 1989), but it is tedious, time consuming, costly and needs large volumes toxic and 
flammable solvents. SPME has drawbacks of high cost, sample carry-over, and a decline in performance with time. LPME produces some obvious disadvantages: solvent drops are ready to break, air bubble is tending to form, time is long and sometimes equilibrium is not achieved easily in short time (Melwanki \& Fuh, 2008).

SPE has extinguished from many extraction techniques and has been widely used for the concentration and measurement of many pollutants (Halden \& Paull, 2004; Chu \& Metcalfe, 2007; Sapkota et al., 2007). SPE has been an effective sample-handing technique with many obvious advantages such as high recovery, high pre-concentration factors, low consumption of organic solvents, simplicity, easy operation and automation. In SPE the analytes to be extracted are partitioned between a solid and liquid and these analytes must have a greater affinity for the solid phase than for the same matrix, which are better than the two immiscible liquids as in LLE. The different mechanisms of retention or elution are due to inter-molecular forces between the analytes, the active sites on the surface of the adsorbent and liquid phase or matrix. Therefore, the types of the adsorbents are the key to SPE process.

The silicon dioxide/polystyrene composite microspheres $\left(\mathrm{SiO}_{2} /\right.$ PS) is a mixed-mode sorbent which was prepared through polymerization of styrene on the surface of $\mathrm{SiO}_{2}$. It has both a hydrophilicity of $\mathrm{SiO}_{2}$ and a lipophilicity of polystyrene, and was successfully used to extract nitrophenol and alkylphenol from aqueous environmental samples (Shen S. C. et al., 2012).

Sensitive and selective approaches for determination of TCC, TCS and MTCS have been established, including GC/MS (Coogan et al., 2007; Kawaguchi et al., 2008)], GC/MS/MS (Boehmer et al., 2004), LC/MS (Chu \& Metcalfe, 2007; Sapkota et al., 2007) and LC/MS/MS (Zhao et al., 2011; Shen J. et al., 2012). In order to reduce complex derivatization step and possible interferences of other compounds, HPLC-ESI-MS was selected for the sensitive determination of TCC, TCS and MTCS because they are compounds with strong polarity.

The aim of this work is to develop a rapid SPE-UHPLC-MS method for simultaneous determination of TCC, TCS and MTCS in environmental water samples. To accomplish its application in environmental monitoring of environmental water, silicon dioxide/polystyrene $\left(\mathrm{SiO}_{2} / \mathrm{PS}\right) \mathrm{SPE}$ cartridges was selected for the pre-concentrations. A series of key parameters influencing the enrichment efficiency of TCC, TCS and MTCS have been investigated and optimized. It could provide enough valid evidence for post-study.

\section{Materials and Methods}

\section{Materials}

HPLC-grade methanol, acetonitrile, and acetone were purchased from Tedia Company Inc. (Fairfield, Ohio, USA). TCC were purchased from Dajie Technical Co. Ltd. (Hunan, China). TCS and MTCS were obtained from Dr. Ehrenstorfer (Augsburg, Germany). Standard stock solution of TCC, TCS and MTCS (100 mg.L) was prepared in methanol and then stored at $4^{\circ} \mathrm{C}$. Fresh working solutions were prepared daily by diluting the stock solution with purified water. The $\mathrm{SiO}_{2} / \mathrm{PS}$ sorbent (5 $\mu \mathrm{m})$ was obtained from Lantian Pharmaceutical co. Ltd. (Wuhan, China).

A $\mathrm{SiO}_{2} / \mathrm{PS}$ packed cartridge was prepared by modifying an Agilent cleanert ODS $\mathrm{C}_{18}$ cartridge $(70 \mathrm{~mm} \times 14 \mathrm{~mm}, 6 \mathrm{~mL}$, polypropylene), which was purchased from Agilent Techno- logies (Palo Alto, CA,USA). $500 \mathrm{mg}$ of $\mathrm{SiO}_{2} / \mathrm{PS}$ sorbent was packed into the cartridge, after the C18 packing was evacuated. The polypropylene upper frit and lower frit were placed at each end of the cartridge to hold the $\mathrm{SiO}_{2} / \mathrm{PS}$ packing fixed. Then the bottom end of the cartridge was connected to a SHBIII vacuum pump (Great Wall Scientific and Trade Co. Ltd., Zhengzhou, Henan), and the upper end of cartridge was connected with a PTFE suction tube, the other end of which was immersed into the water sample. The whole SPE device was washed by sufficient methanol and purified water before the first usage, in order to avoid the pollution of organic contaminants.

\section{Instruments}

The high-performance liquid chromatography-mass spectrometry equipment was an Agilent 1100 LC system, including an electrospray ionization mass spectrometer (ESI-MS), a quaternary pump, a column thermostat, and an automatic sample injector with a $100 \mu \mathrm{L}$ loop. A personal computer equipped with the Agilent ChemStation program for HPLC-MS was used to process the chromatographic data. An Agilent Eclipse $\mathrm{XDB}^{-\mathrm{C}_{8}}$ column, $4.6 \mathrm{~mm} \times 150 \mathrm{~mm}, 5 \mu \mathrm{m}$ particle size, was used to held at $30^{\circ} \mathrm{C}$ to analyze TCS. The injected sample volume was $10 \mu \mathrm{L}$, and the binary mobile phase was composed of $25 \%$ water and $75 \%$ methanol $(\mathrm{v} / \mathrm{v})$ at a constant flow rate of .8 $\mathrm{mL} / \mathrm{min}$. Under these chromatographic conditions, one HPLCMS run can be finished within $10 \mathrm{~min}$. MS conditions were maintained as follows: drying gas $10.0 \mathrm{~L} / \mathrm{min}$; nebulizer pressure $30 \mathrm{psi}$; chemcur. $.32 \mu \mathrm{A}$; quadratic temperature $99^{\circ} \mathrm{C}$; high vac. $1.1 \times 10^{-5}$ Torr. Target compounds analysis was carried out in negative ionization mode and quantification was performed under SIM conditions.

\section{SPE Procedure}

The cartridge packed with $\mathrm{SiO}_{2} / \mathrm{PS}$ adsorbent was conditioned with $10 \mathrm{~mL}$ of methanol in acetone (50\%), followed by 5 $\mathrm{mL}$ of methanol and $5 \mathrm{~mL}$ of water before use. Aliquots (300 $\mathrm{mL}$ ) of water sample were passed through the cartridges at a flow rate of approximately $10 \mathrm{~mL} / \mathrm{min}$. Following the loading of the sample, the cartridge was eluted with $5 \mathrm{~mL}$ hexane, and 5 $\mathrm{mL}$ dichloromethane to remove the co-adsorbed matrix substance. After the cartridge was dried with a vacuum, $2 \times 5 \mathrm{~mL}$ of $5 \%$ methanol in water was used to wash it. Then the cartridge was dried under vacuum for $10 \mathrm{~min}$. The cartridge was eluted with $3 \times 5 \mathrm{~mL}$ methanol in acetone (50\%) at a slow rate of $1 \mathrm{~mL} / \mathrm{min}$. The combined extract was dried under $\mathrm{N}_{2}$ gas and then reconstituted in $.5 \mathrm{~mL}$ of methanol.

\section{Results and Discussion}

\section{Method Development}

\section{Selection of the Adsorbents}

SPE has gained popularity as a technique for the extraction of polar to medium-polarity analytes from aqueous environmental samples. With a number of different sorbents applied in SPE and all kinds of commercial SPE cartridges emerged in the market, SPE can almost meet all the demands of preconcentrations and clean-up in the environmental monitoring. The recoveries of TCC, TCS and MTCS were investigated by using four different SPE cartridges: Agilent SampliQ Optimised Polymer technology (OPT), Agela Cleanert PEP, bamboo-activated char- 
coal (BAC) and $\mathrm{SiO}_{2} / \mathrm{PS}$ with eluate of $50 \%$ methanol in acetone. As shown in Figure 1, the experimental results indicate that the OPT sorbent is unsuitable for the isolation of TCC, TCS and MTCS because of its poor recoveries. The Agela Cleanert PEP and the BAC sorbents showed efficient selective adsorption for TCC, TCS and MTCS, respectively. However, these SPE cartridges are unsuitable for the simultaneous determination of all three analytes. Only the $\mathrm{SiO}_{2} / \mathrm{PS}$ sorbent showed satisfactory recoveries for TCC, TCS and MTCS and was thus selected to be the SPE material in this study.

\section{Selection of the Eluant and Its Volume}

Eluant is a key parameter that affects the recovery of target compounds. Three eluants, including methanol, acetone, and $50 \%$ methanol in acetone, were tested. The results indicate that $50 \%$ methanol in acetone has better desorption efficiencies (the recoveries were $91.2 \%, 94.5 \%$ and $96.0 \%$ for TCC, TCS and MTCS, respectively), as shown in Figure 2. Therefore, 50\% methanol in acetone was chosen as the eluant.

Then the effect of eluant volume which directly affects the recovery of analytes was investigated. A series of experiments were designed and performed by changing the volumes of eluant over the range of $2.3-15.0 \mathrm{~mL}$. The results showed that the recoveries TCC, TCS and MTCS increased with the increase of the eluant volume between $3.0 \mathrm{~mL}$ and $9.0 \mathrm{~mL}$ (Figure 3). When the volume is more than $9.0 \mathrm{~mL}$, the extraction efficiencies of TCC, TCS and MTCS almost kept constant. Therefore, $9.0 \mathrm{~mL}$ eluant was used as the eluant in all subsequent experiments.

\section{Influence of $\mathbf{p H}$}

To examine the effect of the $\mathrm{pH}$ of the water sample on the

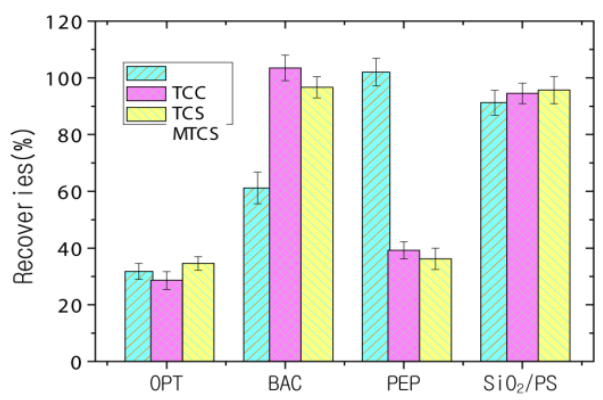

\section{Figure 1.}

Effect of adsorbents on the recoveries of TCC, TCS and MTCS.

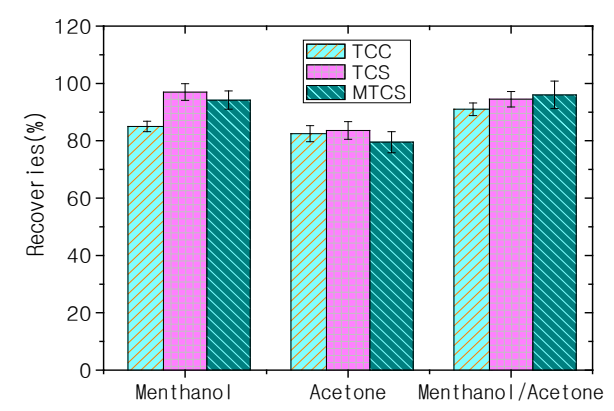

Figure 2.

Effect of eluant on the recoveries of TCC, TCS and MTCS. recoveries of TCC, TCS and MTCS, a series of experiments were performed by changing the $\mathrm{pH}$ of the water samples from 2.0 to 13.0, as shown in Figure 4. The result indicates that the recoveries of TCC, TCS and MTCS maintain high from $\mathrm{pH} 2$ to $\mathrm{pH} 10$. However, under the condition of alkalinity, the recovery of TCC kept satisfactory while that of TCS and MTCS dropped dramatically. This result may possibly be attributed to the structural characteristics of TCS. Strong alkalinity may result in the deprotonation of the hydroxyl of TCS and MTCS, which affects their solubility in water. According to these experimental results, the $\mathrm{pH}$ of solutions was adjusted in the range from 4.0 to 6.0 in the subsequent experiments.

\section{Influence of Flow Rate}

The flow rate of water sample affects the recovery of TCS and controls the sample pretreatment time. Here, the flow rates of the sample were optimized in the range of $1.0-3.0 \mathrm{~mL} / \mathrm{min}$ with the limitation on the negative pressure of the pump. The results from Figure 5 show that the flow rate had no obvious influence on the extraction efficiency of TCC, TCS and MTCS. In order to save extraction time, the highest flow rate, 3.0 $\mathrm{mL} / \mathrm{min}$, was adopted in the following experiments

\section{Method Validation}

Under the given optimal conditions, a number of important parameters of the method, such as linearity range, correlation coefficient $\left(r^{2}\right)$, reproducibility, limit of detection, and recovery, were studied. Linearity was tested by varying the concentrations of TCC, TCS and MTCS from $5 \mathrm{ng} / \mathrm{L}$ to 20,000 ng/L,

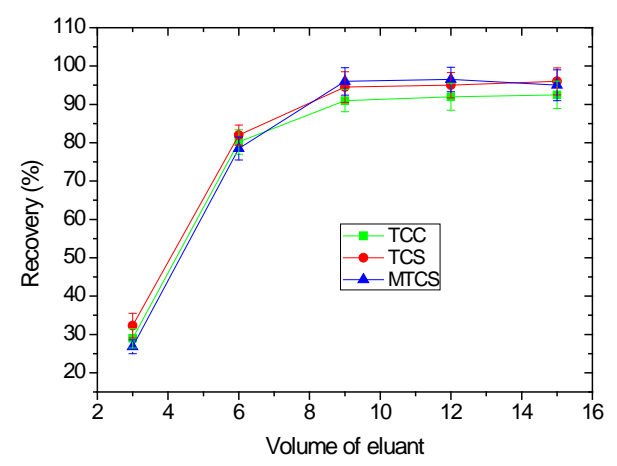

Figure 3.

Effect of volume of eluant on the recoveries of TCC, TCS and MTCS.

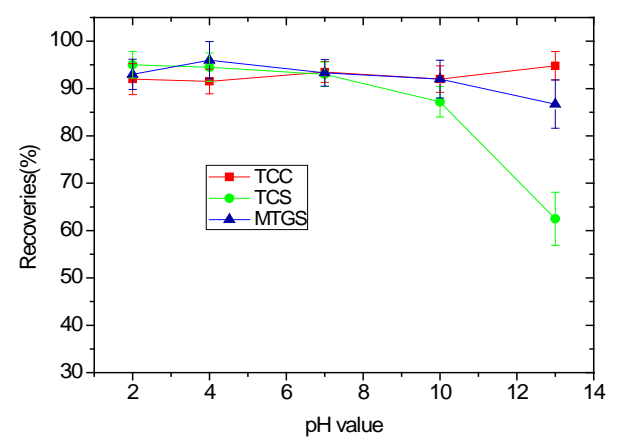

Figure 4.

Effect of $\mathrm{pH}$ of samples on the recoveries of TCC, TCS and MTCS. 


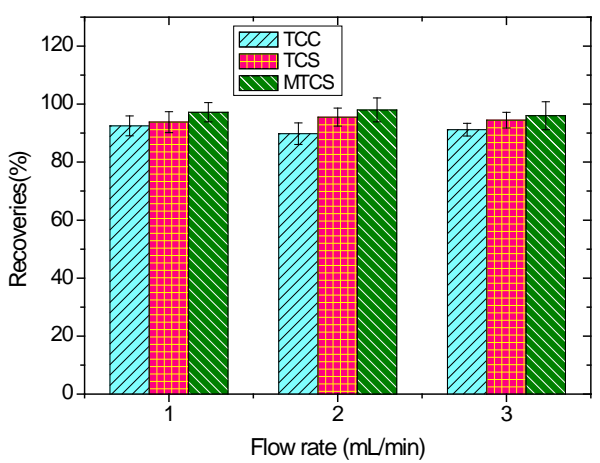

Figure 5.

Effect of flow rate on the recoveries of TCC, TCS and MTCS.

with $r^{2}>$.99. Limits of detection (LOD) of the analytes were calculated with signal/noise ratios $(\mathrm{S} / \mathrm{N})$ of 3 . Limits of quantification (LQD) were calculated with signal/noise ratios $(\mathrm{S} / \mathrm{N})$ of 10. Detailed information on the limits of the analytes was provided in Table 1.

To investigate the effects of the sample matrix, two water samples collected from Xiaoqing River were spiked with TCC, TCS and MTCS at different levels and were then analyzed. The recoveries of spiked water are listed in Table 2 . The effects of the matrix on the signal were insignificant after sample cleanup by using SPE.

\section{Determination of TCC, TCS and MTCS in Environmental Water Samples}

For a preliminary survey and for method validation, the method developed was used for simultaneous analysis of the three target compounds in a variety of environmental water samples. Three river water samples were collected in Xiaoqing Rivers in Jinan area, Shandong Province, China. The sample 1\# was collected in the upstream of the wastewater treatment plant, $.5 \mathrm{~km}$ from the discharge port. The sample $2 \#$ was collected in the downstream of wastewater treatment plant near the discharge port, and samples $3 \#$ was collected downstream of wastewater treatment plant, apart from the discharge port $2 \mathrm{~km}$. Two sea water samples collected in Yellow Sea at Qingdao port. Sample $1 \#$ was from the coast, and sample $2 \#$ was $2 \mathrm{~km}$ apart from the coast. Figure 6 shows a typical HPLC-ESI-MS chromatogram obtained from a river water extract. The average concentrations of the target compounds in a variety of environmental water samples are listed in Table $\mathbf{3 .}$

\section{Conclusion}

A procedure for the determination of TCC, TCS and MTCS in environmental water samples is developed. The analytes were simultaneously isolated using silicon dioxide/polystyrene composite microspheres SPE sorbent, and then they were determined with UHPLC-ESI-MS. Under the optimized conditions, the LODs were $1.0 \mathrm{ng} / \mathrm{L}, 2.5 \mathrm{ng} / \mathrm{L}$ and $4.5 \mathrm{ng} / \mathrm{L}$ for TCC, TCS and MTCS, respectively. The spiked recoveries of TCC, TCS and MTCS in environmental water samples were achieved in the range of $89.5 \%$ - $96.8 \%$ with RSD below $5.7 \%$. With this method, the TCC, TCS and MTCS in a variety of environmental water samples from Xiaoqing Rivers and Yellow Sea were detected. This work indicates that the proposed method is con-
Table 1.

Linear range and limits of detection of the method.

\begin{tabular}{ccccc}
\hline Analyte & Liner range $(\mathrm{ng} / \mathrm{L})$ & $\mathrm{r}^{2}$ & LOD & LOQ \\
\hline TCC & $5-2000$ & .996 & 1.0 & 3.5 \\
TCS & $5-2000$ & .994 & 2.5 & 8.0 \\
MTCS & $5-2000$ & .997 & 4.5 & 14.0 \\
\hline
\end{tabular}

Table 2.

Experimental recoveries of TCC, TCS and MTCS from spiked real water samples.

\begin{tabular}{ccccc}
\hline Analyte & Found (ng/L) & Added (ng/L) & Recovery (\%) & RSD (\%) \\
\hline TCC & 42 & 50 & 89.5 & 5.2 \\
& 136 & 200 & 93.4 & 3.8 \\
TCS & 62 & 50 & 93.8 & 4.9 \\
& 93 & 200 & 95.6 & 5.7 \\
MTCS & ND $^{\mathrm{a}}$ & 50 & 96.8 & 3.3 \\
& ND & 200 & 94.2 & 3.9 \\
\hline
\end{tabular}

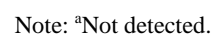

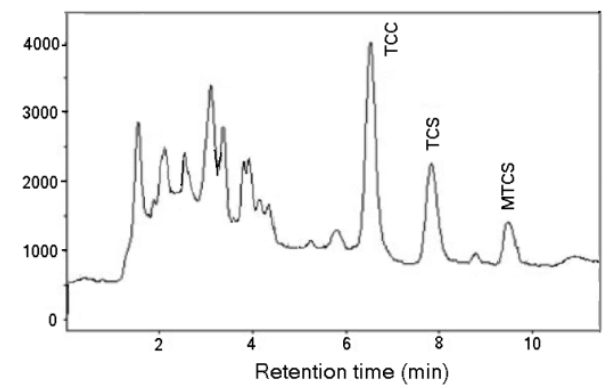

Figure 6.

Typical HPLC-ESI-MS chromatogram obtained from river water.

Table 3.

Analytical results of TCC, TCS and MTCS in environmental water samples.

\begin{tabular}{cccc}
\hline SAmples & TCC (ng/L) & TCS (ng/L) & MTCS (ng/L) \\
\hline Xiaoqing River & & & \\
$1 \#$ & 42 & 62 & $\mathrm{ND}^{\mathrm{a}}$ \\
$2 \#$ & 326 & 245 & 22 \\
$3 \#$ & 136 & 93 & $\mathrm{ND}$ \\
Sea water & & & \\
$1 \#$ & 28 & 71 & 18 \\
$2 \#$ & $\mathrm{ND}$ & 12 & $\mathrm{ND}$ \\
\hline
\end{tabular}

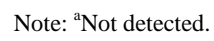

venient and reliable for the determination of TCC, TCS and MTCS in environmental water samples. This method could be applied to monitor TCC, TCS in various environmental water samples.

\section{Acknowledgements}

The authors are thankful to the financially supports by the National Natural Science Foundation of China (No. 21077069).

\section{REFERENCES}

Ahn, K. C., Zhao, B., Chen, J., Cheredenichenko, G., Sanmarti, E., \& Denision, M. S. (2008). In vitro biological activities of the antimicrobials triclocarban, its analogues, and triclosan in bioassay screens: Receptorbased bioassay screens. Environmental Health Perspectives, $116,1203-1210$. 
http://dx.doi.org/10.1289/ehp.11200

Boehmer, W., Ruedel, H., Wenzel, A., \& Schroeter-Kermani, C. (2004). Retrospective monitoring of triclosan and methyl-triclosan in fish: Results from the German environmental specimen bank. Organohalogen Compounds, 66, 1516-1521.

Canosa, P., Pérez-Palacios, D., Garrido-López, A., Tena, T. M., Rodríguez, I., Rubi, E., \& Cela, R. (2007). Pressurized liquid extraction with in-cell clean-up followed by gas chromatography-tandem mass spectrometry for the selective determination of parabens and triclosan in indoor dust. Journal of Chromatography A, 1161, 105-112. http://dx.doi.org/10.1016/j.chroma.2007.05.089

Chalew, T. E. A., \& Halden, R. U. (2009). Environmental exposure of aquatic and terrestrial biota to triclosan and triclocarban. The Journal of the American Water Resources Association, 45, 4-13. http://dx.doi.org/10.1111/j.1752-1688.2008.00284.x

Coogan, M. A., Edziyie, R. E., La Point, T. W., \& Venable, B. J. (2007). Algal bioaccumulation of triclocarban, triclosan, and methyl-tri- closan in a North Texas wastewater treatment plant receiving stream. Chemosphere, 67, 1911-1918. http://dx.doi.org/10.1016/j.chemosphere.2006.12.027

Coogan, M. A., \& La Point, T. W. (2008). Snail bioaccumulation of triclocarban, triclosan, and methyltriclosan in a north texas, USA, stream affected by wastewater treatment plant runoff. Environmental Toxicology and Chemistry, 27, 1788-1793. http://dx.doi.org/10.1897/07-374.1

Chu, S., \& Metcalfe, C. D. (2007). Simultaneous determination of triclocarban and triclosan in municipal biosolids by liquid chromatography tandem mass spectrometry. Journal of Chromatography A, 1164, 212-218. http://dx.doi.org/10.1016/j.chroma.2007.07.024

Guo, J. H., Li, X. H., Cao, X. L., Li, Y., Wang, X. Z., \& Xu, X. B. (2009). Determination of triclosan, triclocarban and methyl-triclosan in aqueous samples by dispersive liquid-liquid microextraction combined with rapid liquid chromatography. Journal of Chromatography A, 1216, 3038-3043. http://dx.doi.org/10.1016/j.chroma.2009.02.021

Halden, R. U., \& Paull, D. H. (2004). Analysis of triclocarban in aquatic samples by liquid chromatography electrospray ionization mass spectrometry. Environmental Science and Technology, 38, 48494855. http://dx.doi.org/10.1021/es049524f

Halden, R. U., \& Paull, D. H. (2005). Co-occurrence of triclocarban and triclosan in US water resources. Environmental Science and Technology, 39, 1420-142. http://dx.doi.org/10.1021/es049071e

Hinther, A., Bromba, C. M., Wulff, J. E., \& Helbing, C. C. (2011). Effects of triclocarban, triclosan, and methyl triclosan on thyroid hormone action and stress in frog and mammalian culture systems. Environmental Science and Technology, 45, 5395-5402. http://dx.doi.org/10.1021/es1041942

Kawaguchi, M., Ito, R., Honda, H., Endo, N., Okanouchi, N., Saito, K., Seto, Y., \& Nakazawa, H. (2008). Stir bar sorptive extraction and thermal desorption-gas chromatography-mass spectrometry for trace analysis of triclosan in water sample. Journal of Chromatography A, 1206, 196-199. http://dx.doi.org/10.1016/j.chroma.2008.08.060

Liska, I., Krupcik, J., \& Leclercp, P. A. (1989). The use of solid sorbents for direct accumulation of organic compounds from water matrices-a review of solid-phase extraction techniques. Journal of High
Resolution Chromatography, 12, 577-590.

http://dx.doi.org/10.1002/jhrc.1240120903

Melwanki, M. B., \& Fuh, M. R. (2008). Dispersive liquid-liquid microextraction combined with semi-automated in-syringe back extraction as a new approach for the sample preparation of ionizable organic compounds prior to liquid chromatography. Journal of Chromatography A, 1198, 1-6. http://dx.doi.org/10.1016/j.chroma.2008.05.007

Mezcua, M., Gomez, M. J., Ferrer, I., Aguera, A., Hernando, M. D., \& Fernandez-Alba, A. R. (2004). Evidence of 2,7/2,8-dibenzodichlorop-dioxin as a photodegradation product of triclosan in water and wastewater samples. Analytica Chimica Acta, 524, 241-247. http://dx.doi.org/10.1016/j.aca.2004.05.050

Montes, R., Rodríguez, I., Rubí, E., \& Cela, R. (2005). Optimization of solid-phase microextraction conditions for the determination of triclosan and possible related compounds in water samples. Journal of Chromatography A, 1072, 107-115. http://dx.doi.org/10.1016/j.chroma.2004.11.032

Orvos, D. R., Versteeg, D. J., Inauen, J., Capdeveille, M., Rothenstein A., \& Cunningham, V. (2002). Aquatic toxicity of triclosan. Environmental Toxicology and Chemistry, 21, 1338-1349. http://dx.doi.org/10.1002/etc.5620210703

Ponte, C., Richard, J., Bonte, C., Lequien, P., \& Lacombe, A. (1974). Methemoglobinemia in Newborn-Discussion of Etiological Role of Trichlorocarbanilide. Semaine Des Hopitaux, 50, 359-365.

Sapkota, A., Heidler, J., \& Halden, R. U. (2007). Detection of triclocarban and two co-contaminating chlorocarbanilides in US aquatic environments using isotope dilution liquid chromatography tandem mass spectrometry. Environmental Research, 103, 21-29. http://dx.doi.org/10.1016/j.envres.2006.03.006

Shen, J. Y., Chang, M. S., Yang, S. H., \& Wu, G. J. (2012). Simultaneous determination of triclosan, triclocarban, and transformation products of triclocarban in aqueous samples using solid-phase micro-extraction-HPLC-MS/MS. Journal of Separation Science, 35, 2544-2552. http://dx.doi.org/10.1002/jssc.201200181

Shen, S. C., Bi, J. N., Sui, L. L., \& Liu, Y. H, (2012). Preparation of $\mathrm{SiO}_{2} / \mathrm{PS}$ composite microsphere and its application as solid-phase extraction sorbent. Chinese Journal of Analysis Laboratory, 31, 51-54.

Sun, J., Yi, C. L., Zhao, R. S., Wang, X., Jiang, W. Q., \& Wang, X. K. (2012). Determination of trace triclosan in environmental water by microporous bamboo-activated charcoal solid-phase extraction combined with HPLC-ESI-MS. Journal of Separation Science, 35, 27812786. http://dx.doi.org/10.1002/jssc.201200328

Zhao, R., Cheng, C., Yuan, J., Jiang, T., Wang, X., \& Lin, J. (2007). Sensitive measurement of ultratrace phenols in natural water by purge-and-trap with in situ acetylation coupled with gas chromatography-mass spectrometry. Analytical and Bioanalytical Chemistry, 387, 687-694. http://dx.doi.org/10.1007/s00216-006-0890-7

Zhao, R. S., Wang, X., Sun, J., Hu, C., \& Wang, X. K. (2011). Determination of triclosan and triclocarban in environmental water samples with ionic liquid/ionic liquid dispersive liquid-liquid micro-extraction prior to HPLC-ESI-MS/MS. Microchimica Acta, 174, 145-151. http://dx.doi.org/10.1007/s00604-011-0607-2 